\title{
Study of secondary instability of precursor magnetic island in COMPASS density limit disruptions
}

Cite as: Phys. Plasmas 27, 022515 (2020); https://doi.org/10.1063/1.5123677

Submitted: 08 August 2019 . Accepted: 01 January 2020 . Published Online: 19 February 2020

F. Salzedas (D), S. I. W. Shah (D), J. Havlicek (D), J. Stöckel, P. Háček (D), J. Varju, A. Havránek, R. Pánek, and

C. Silva (iD)

\section{AVS Quantum Science}

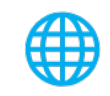




\title{
Study of secondary instability of precursor magnetic island in COMPASS density limit disruptions
}

Cite as: Phys. Plasmas 27, 022515 (2020); doi: 10.1063/1.5123677

Submitted: 8 August 2019 . Accepted: 1 January 2020 .

Published Online: 19 February 2020

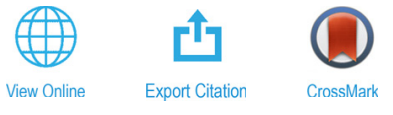

\author{
F. Salzedas, ${ }^{1,2, a)}$ (D) S. I. W. Shah, ${ }^{2,3}$ (D) J. Havlicek, ${ }^{4}$ (D) J. Stöckel, ${ }^{4}$ P. Háček, ${ }^{4}$ (iD J. Varju, ${ }^{4}$ A. Havránek, ${ }^{4}$ R. Pánek, \\ and C. Silva ${ }^{2}$ iD
}

\author{
AFFILIATIONS \\ 'Universidade do Porto, Faculdade de Engenharia, 4200-465 Porto, Portugal \\ ${ }^{2}$ Instituto de Plasmas e Fusão Nuclear, Instituto Superior Técnico, Universidade de Lisboa, 1049-001 Lisboa, Portugal \\ ${ }^{3}$ National Tokamak Fusion Program, Islamabad 3329, Pakistan \\ ${ }^{4}$ Institute of Plasma Physics of the CAS, Za Slovankou 3, 182 00, Prague 8, Czech Republic

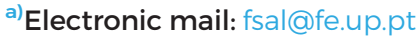

\begin{abstract}
A systematic study of COMPASS density limit disruptions (DLDs) was carried out to analyze the relation between the precursor magnetic island and its secondary instability (SI). In all of these plasmas, the SI was always found present at the onset of energy confinement erosion. The duration of this erosion was shorter for precursor quasi-locked island than for rotating island, and the larger the island amplitude, the shorter the duration. It was also found, at the onset of the erosion of energy confinement, an inverse relation between the magnetic island amplitude and its rotation frequency, showing that erosion can start at different values of precursor island amplitude, depending on what is its rotation frequency. DLDs occur either with smaller precursor island rotating faster or larger island rotating slower (quasi-locked).
\end{abstract}

Published under license by AIP Publishing. https://doi.org/10.1063/1.5123677

\section{INTRODUCTION}

It is well known that density limit disruptions (DLDs) restrict the operation of large tokamaks like ITER in high density plasmas, which are able to cause serious structural damage in the machine. ${ }^{1}$ Improving the knowledge on DLDs dynamics can provide better strategies to ameliorate or avoid them. The abrupt destruction of the energy confinement marks the onset of the disruption in a phase termed as the thermal quench (TQ). This is swiftly followed by a rapid decay of the plasma current, termed as the current quench (CQ) phase. In plasmas with an edge safety factor $q_{95} \approx 3-4$, DLDs are typically preceded by a resistive MHD mode with poloidal $(m)$ and toroidal $(n)$ mode numbers $m=2$ and $n=1$, respectively. The exact beginning of the thermal quench cannot yet be predicted, but it is observed to take place at some stage of the non-linear development of this precursor magnetic island when its rotation frequency is decreasing or is already very low. During the TQ, the enhanced heat transport in the radial direction has been usually attributed to stochastization of the field lines, due to overlapping of magnetic islands. ${ }^{1,2}$ Contrary to this hypothesis, a secondary instability (SI) to the magnetic island was observed prior to the TQ, when the amplitude of the magnetic island is large and its rotation frequency is low, first in $\mathrm{JET}^{3}$ and then in COMPASS. $^{4}$

Non-linear MHD numerical simulations have shown that island deformation during its rapid growth can lead to secondary magnetic island formation. ${ }^{6}$ A review ${ }^{7}$ of the theory of current sheet formation that leads to magnetic reconnection mentions the occurrence of plasmoids during magnetic island evolution. The validity ranges of the mentioned numerical works are not directly comparable to experimental conditions. So one cannot claim with certainty that the SI observed in JET and in COMPASS DLDs, here reported, is the same as observed in those numerical simulations, despite some qualitative similarities between them. Also so far we have only carried on these studies on disruptions of the type density limit.

\section{A. Secondary instability in JET DLDs}

In the $\mathrm{JET}^{3}$ study, DLDs were provoked using a Neon gas puff on Deuterium (D) plasmas to keep the density limit below the cut-off frequency of the electron cyclotron emission radiometer (ECE). The 
correlation observed between the perturbations caused by the SI in the magnetic poloidal flux and in the electron temperature, measured using the ECE radiometer, was very high, indicating that the SI came from the same position as the magnetic island. Moreover, the ECE allowed determining that the presence of the SI to the magnetic island was associated with erosion of energy confinement, namely the occurrence of minor disruptions, that precede the major disruption, as well as with the major disruption itself. The SI was characterized by small amplitude perturbations, superimposed on the perturbations of the underlying $2 / 1$ mode. They were observed when the rotation of the magnetic island decreases prior to the disruption.

In this phase, the island evolves non-linearly, and $\dot{B}_{\theta}$ oscillations become non-harmonic. The frequency of the SI small amplitude perturbations is higher and has a larger bandwidth than the rotation frequency of the 2/1 mode. Another relevant observed feature was that no toroidal or poloidal mode numbers could be assigned to the SI. These characteristics of the SI can be measured using magnetic coils available in all tokamaks.

\section{B. Study of the secondary instability in COMPASS DLDS}

Following these observations, ${ }^{3}$ we wanted to find if the SI has any particular relation with the $2 / 1$ magnetic island, since it is always observed only when a large island experiences the typical decrease in rotation frequency that precedes the disruption, and if a DLD is always preceded by SI, and how long does it last. To answer these questions, we focused mainly on the analysis of magnetic field perturbations, from two Mirnov coils arrays toroidally apart by $180^{\circ}$ and four saddle loop coils (coil number 22) in the low field side (LFS), each spanning $90^{\circ}$ in the toroidal direction.

For this purpose, a systematic set of DLDs was provoked primarily on single null Ohmic D-shaped plasmas by continuous Deuterium gas puff on a Deuterium plasma in the following range of plasma currents and toroidal magnetic fields. ${ }^{5}$ The plasma current $I_{p}$, ranged from $140 \mathrm{kA} \leq I_{p} \leq 230 \mathrm{kA}$ at a toroidal magnetic field of $0.92 \mathrm{~T} \leq$ $B_{\phi} \leq 1.38 \mathrm{~T}$ resulting in a slightly variable range of edge safety factor $3.7 \leq q_{95} \leq 4.4$. The majority of the discharges were performed for the standard orientation of the plasma current and toroidal field, both are counterclockwise when viewed from above the tokamak.

In a small number of plasmas, other parameters were changed, such as Deuterium gas puff was substituted by Neon gas puff, Neutral Beam Injection (NBI) was used, the plasma current was reversed, and in a couple of plasmas, the carbon limiter was used instead of the usual divertor configuration. In all of these DLDs, the SI was always observed just before the onset of the disruption. It is noteworthy to stress this point because JET and COMPASS are two tokamaks with very distinct sizes, having the major radius of $2.96 \mathrm{~m}$ and $0.56 \mathrm{~m}$, respectively. Moreover, COMPASS has ITER like geometry (1/10 of the ITER plasmas in every dimension) with minor radius for limiter plasmas $a=0.20 \mathrm{~m}$ and for diverted plasmas varying in the range of $0.17 \mathrm{~m} \leq a \leq 0.18 \mathrm{~m}$ with elongation $\kappa=1.8$. So together with JET observations, the experimental evidence gathered in this study firmly points out that the SI should be present in tokamaks of all sizes, most likely including ITER.

The structure of this paper is as follows. In Sec. II, the topology of the underlying MHD mode will be determined. Section III will address the questions raised on the nature of the SI. We draw conclusions and summarize the results in Sec. IV.

\section{MHD MODE IN THE DISRUPTION PRECURSOR}

All the disruptions here studied were preceded by a 2/1 magnetic island that has a rotation frequency of $\approx 15 \mathrm{kHz}$ when its amplitude is small, but in most of the cases, still it had a rotation frequency of $\gtrsim 2$ $\mathrm{kHz}$ at the onset of the disruption. Determining the topology of the magnetic island will also show the different nature of the SI. Figure 1 illustrates two of the typical cases observed, namely pulse \#12748 where DLD was preceded by a rotating mode and pulse \#8945 by a quasi-locked mode. We distinguish these two cases because in the last one, the mode rotation frequency experiences a sudden and significant decrease just before the time $t_{T R}$, defined below, with its value falling by half or more. However, because complete mode stop is not observed, we call this mode quasi-locked.

The mode topology is found from the time evolution of $\dot{B}_{\theta}$ in two Mirnov coils arrays, A and C, as shown in Figs. 1(a) and 1(b). To facilitate the analysis of the signals' relative phases, their amplitudes were standardized (see figures shown in the supplementary material with normal amplitudes),

$$
\frac{\dot{B}_{\theta}-\overline{\dot{B}}_{\theta}}{\sigma_{\dot{B}_{\theta}}},
$$

where $\bar{B}_{\theta}$ is the average of $\dot{B}_{\theta}$ in the first $0.5 \mathrm{~ms}$ of the displayed time window and $\sigma_{\dot{B}_{\theta}}$ is the corresponding standard deviation. Due to this procedure, noise from coils with weak signals such as coils 7, 8, and 9 from array A and coils 7 and 9 from array $C$ was enhanced. Moreover, the time origin was set at minimum loop voltage, $t_{\min V \text { loop }}$.

In a cylindrical approximation, the field perturbation is of the type,

$$
\tilde{B}_{\theta}=\tilde{B}_{0} \cos (m \theta-n \phi-2 \pi f t),
$$

where $f$ is the mode rotation frequency. The poloidal (toroidal) mode number $m(n)$ is the number of toroidal (poloidal) turns around the torus to close a field line, and it is also the number of $\mathrm{O}$ or $\mathrm{X}$ points of the magnetic island in a poloidal (toroidal) cross section. So, the topology of this MHD oscillation, visible in all $\dot{B}_{\theta}$ signals, can be inferred from their relative phases. Two coils at the same poloidal position in arrays $\mathrm{A}$ and $\mathrm{C}$ should display signals in phase opposition if the toroidal mode number is $n=1$ just as observed in Figs. 1(a) and 1(b), e.g., at the time, marked by the red dashed line.

For a poloidal mode number $m=2$, two coils in the same array at the equatorial plane should display signals in phase, just like it is observed for coils 1 and 13, e.g., at the first, vertical red dashed line in Fig. 1(a). This line crosses a minimum of the signal of coil 1 (blue line) and progresses vertically (in the poloidal direction). It crosses another minimum at coil 13 in the high field side (HFS) which is poloidally $180^{\circ}$ apart. Alternatively, one can follow the points with the same phase on its way around the array until returning to the same coil, like the red solid line in Fig. 1(a). This is as if a field line was followed around the torus until it closes on itself. The number of oscillations in coil 1 during this time is the value of $m$. The red solid line is not the straight line expected from Eq. (1), but a snake like line due to other effects like toroidicity and plasma shaping that affect the value of the phase angle $\theta .9,10$

The $m / n=2 / 1$ MHD mode is present until $t=0 \mathrm{~ms}$, when the current quench phase of disruption starts, and the magnetic island is destroyed. While this dominant single mode evolves, some features are observed in all plasmas: 

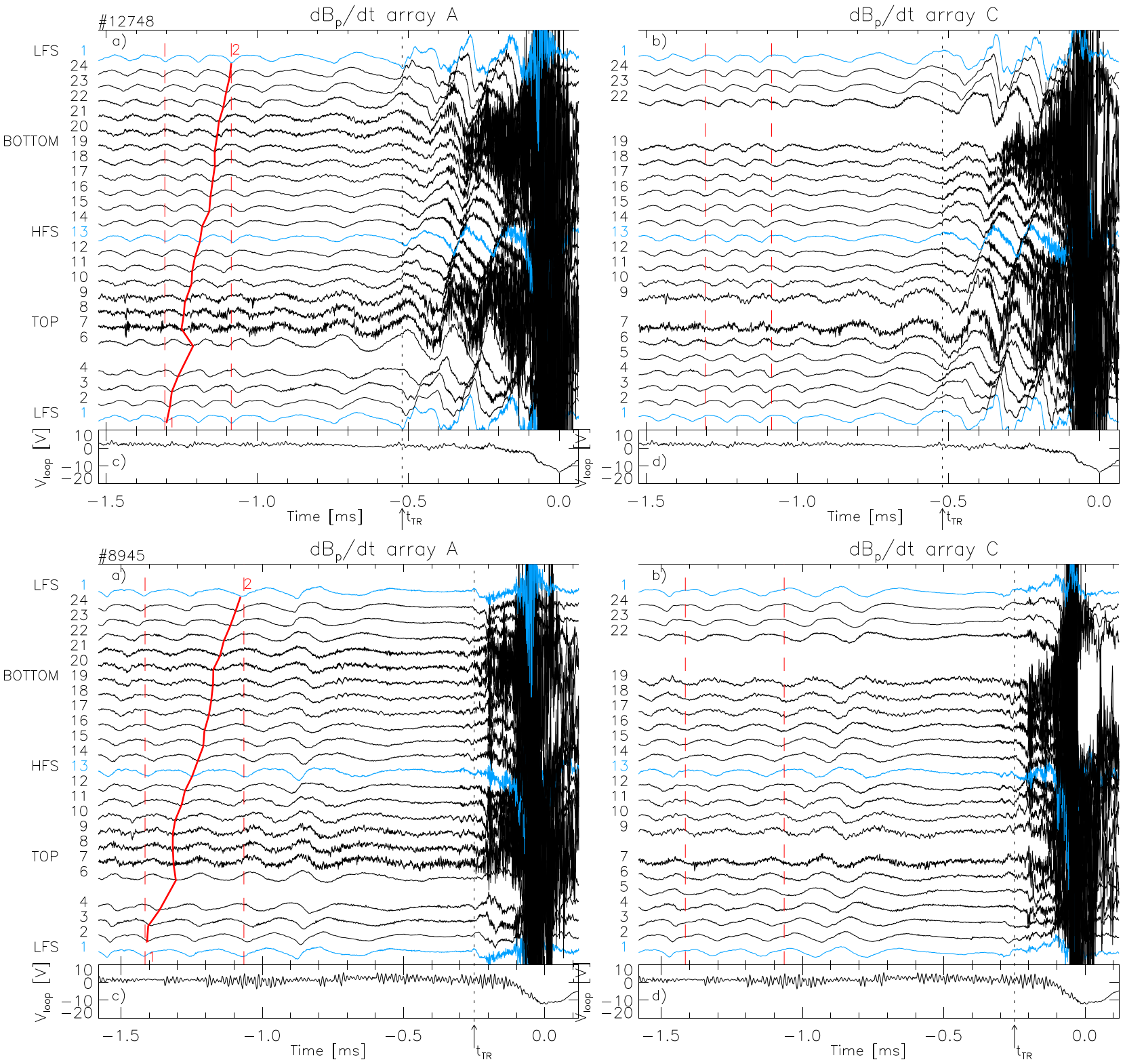

FIG. 1. Time evolution, before the disruption, of $\dot{B}_{\theta}(t)(a)$ and (b), from two Mirnov coils arrays, $A$ and $C$ that are toroidally $180^{\circ}$ apart. The top (bottom) plots are from plasma $\# 12748$ (\#8945) with a rotating (quasi-locked) 2/1 mode preceding the DLD. The coil numbers are indicated at the left, with the respective poloidal position. For each array, the signals are equally spaced. Coils 1 and 13 (blue lines) are in the equatorial plane opposite to each other at the LFS and HFS, respectively. Gaps are due to malfunction/broken coils. (c) and (d) loop voltage. The time axis origin was set at the minimum of the loop voltage. The dotted line at $t_{T R}$ marks the transition to the phase when a secondary instability develops in the magnetic island.

- the mode rotation frequency $f$, decreases as its amplitude increases [see e.g., Figs. 2(d) and 2(f) and Figs. 3(d) and 3(f)]. This is a well-known behavior, being commonly explained ${ }^{11-14}$ by magnetic braking due to the interaction between the mode and its induced currents on the vessel wall, although this mechanism has been questioned. ${ }^{15}$

- when $f$ is decreasing, the mode amplitude deviates from a sinusoidal shape. Such deformation was attributed ${ }^{16}$ to the interaction of the magnetic island with the error field for islands that ended locked to the wall.

- when the mode amplitude starts to become deformed, higher frequency perturbations become visible in all $\dot{B}_{\theta}$ signals. The dotted line labeled $t_{T R}$ in Figs. 1-3 marks this transition to the phase when a secondary instability develops in the magnetic island. An increase in the rotation frequency of the magnetic island is also observed starting just before $t_{T R}$ [see also solid and dashed lines 


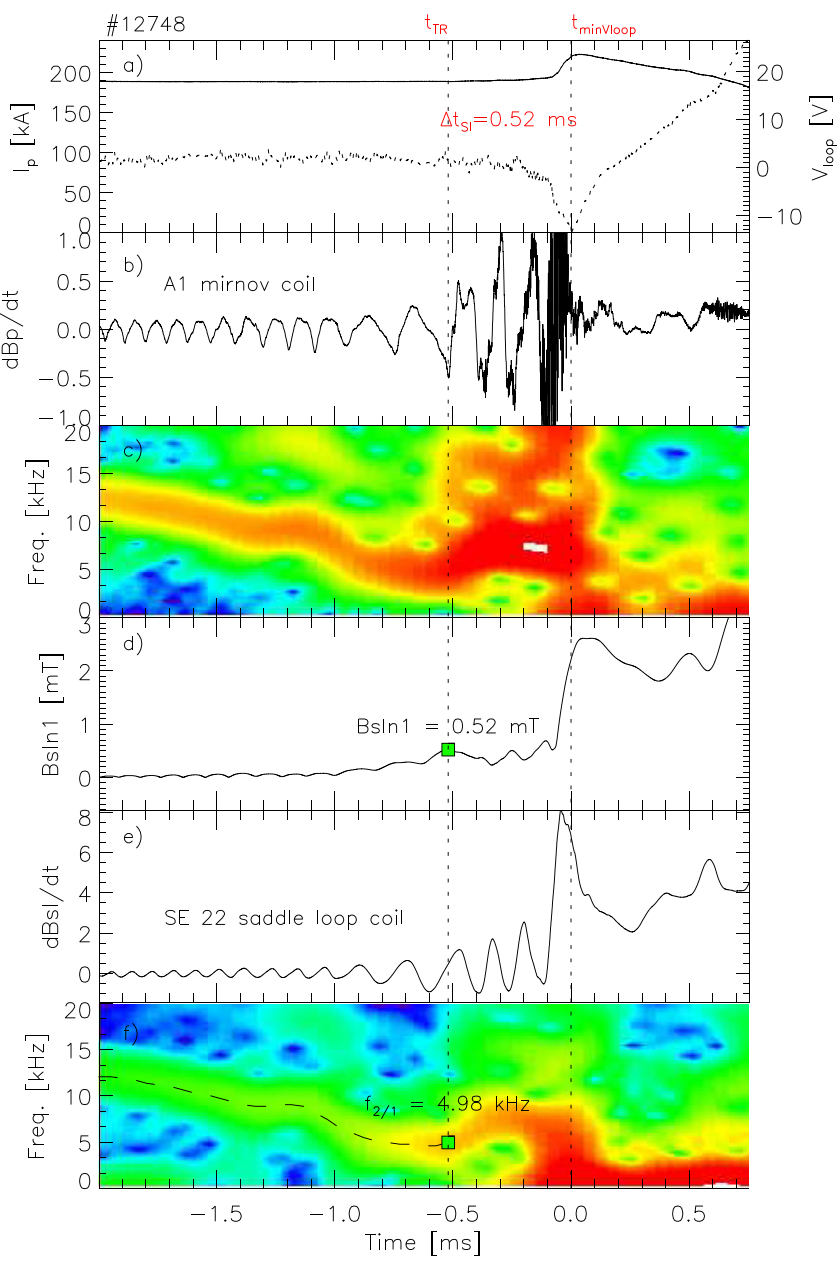

FIG. 2. (a) Plasma current (solid line) and loop voltage (dotted line). (b) $\dot{B}_{p}$ from Mirnov coil array A and corresponding spectrogram (c). (d) Bsln1 derived from four saddle loop coils at the LFS equatorial plane. (e) $\dot{B} s /$ of saddle loop coil SE22 and corresponding spectrogram ( $f$ ). The green squares at (d) and (f) mark the values of $f_{2 / 1}$ and $B s / n 1$ for this plasma, displayed on Figs. 4-6.

in Fig. 5(a)]. Such behavior of the magnetic island seems at odds with the model of resistive magnetic drag because the mode amplitude does not decrease.

\section{THE SECONDARY INSTABILITY TO THE UNDERLYING MHD MODE}

The previous mode number analysis could have been done with the $B_{\theta}$ signals. The reason, $\dot{B}_{\theta}$ was used instead, is because the SI is better seen on $\dot{B}_{\theta}$ than on $B_{\theta}$. A similar analysis, as discussed before, fails to determine a poloidal and toroidal mode numbers of the SI, which is clearly detected in all Mirnov coils. This indicates that the SI has a different topology than the magnetic island. The spectrogram of one of these signals [Fig. 2(c)] shows that the frequency of the SI small amplitude perturbations is higher and has a larger bandwidth than that of the 2/1 mode. These features are consistent with the observations at $\mathrm{JET}^{3}$ where it was inferred from ECE that at the onset of the SI also

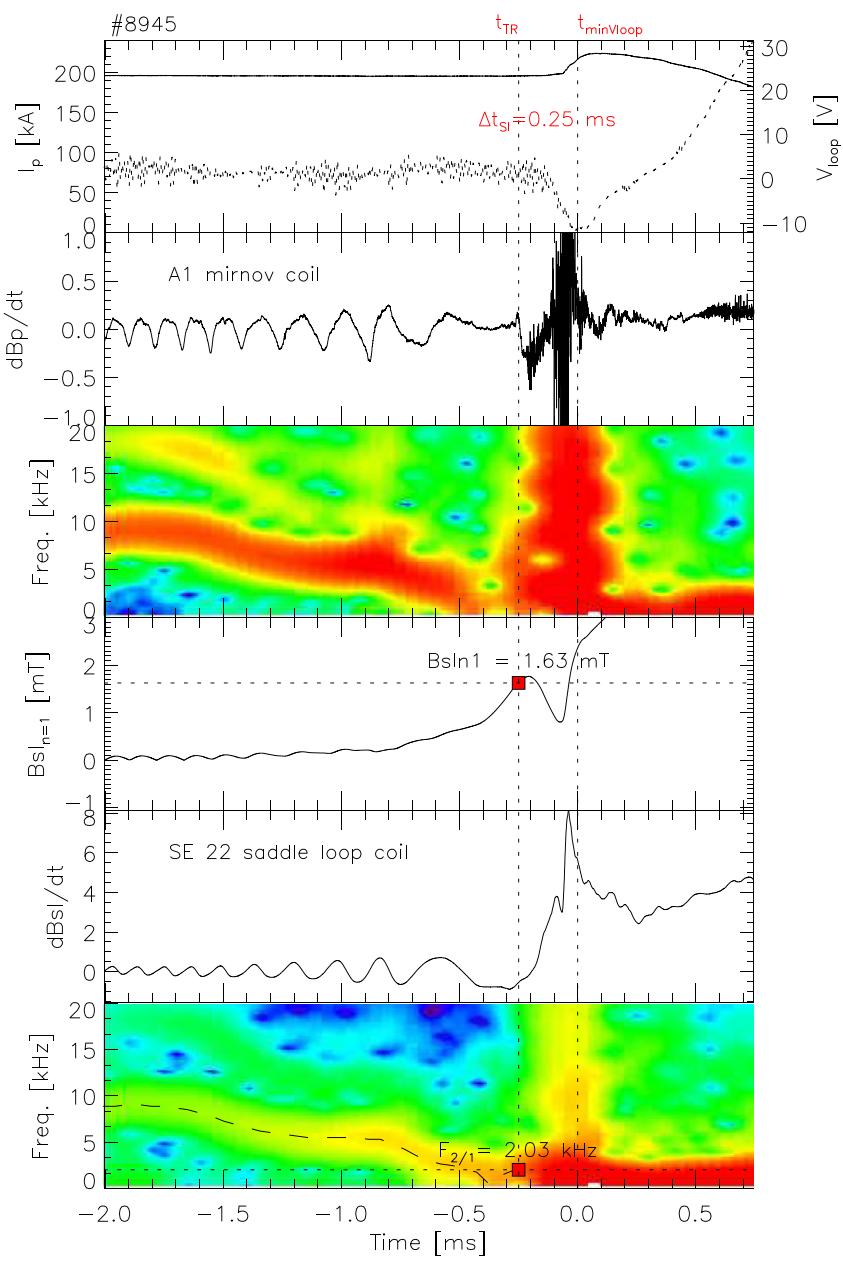

FIG. 3. (a) Plasma current (solid line) and loop voltage (dotted line). (b) $\dot{B}_{p}$ from Mirnov coil array $A$ and the corresponding spectrogram (c). (d) Bsin1 derived from four saddle loop coils at the LFS equatorial plane. (e) Bs/ of saddle loop coil SE22 and corresponding spectrogram ( $f$ ). The red squares at (d) and ( $f$ ) mark the values of $f_{2 / 1}$ and Bs/n1 for this plasma, displayed on Figs. 4-6.

started energy confinement degradation that would become more pronounced as the amplitude and frequency of the SI increased. In COMPASS, this degradation could not be followed with the same time resolution of the magnetic perturbations. But at $t=0$, the loop voltage reaches its minimum value, indicating the TQ is complete.

Figure 4 shows the value of density, energy confinement time, plasma current, and the $n=1$ component of the magnetic field measured by the saddle loop coils positioned at the equator in the LFS (saddle loop coils 22) as a function of the time interval $\Delta t_{S I}=t_{\text {minVloop }}-t_{T R}$. In this period, the SI is active, and energy confinement is strongly degraded, in particular at its end, when the TQ occurs. In the studied cases, $0.24 \mathrm{~ms} \leq \Delta t_{S I} \leq 0.9 \mathrm{~ms}$. The value of $n_{e}$, the maximum attained density in the discharge, does not show any relation with $\Delta t_{S I}$. The same pattern is observed for $\tau_{E}$ and $I_{p}$. However in plasmas with Neon gas puff, $\Delta t_{S I}$ is shorter, as expected; since radiation losses are enhanced in these cases, it drives a faster 

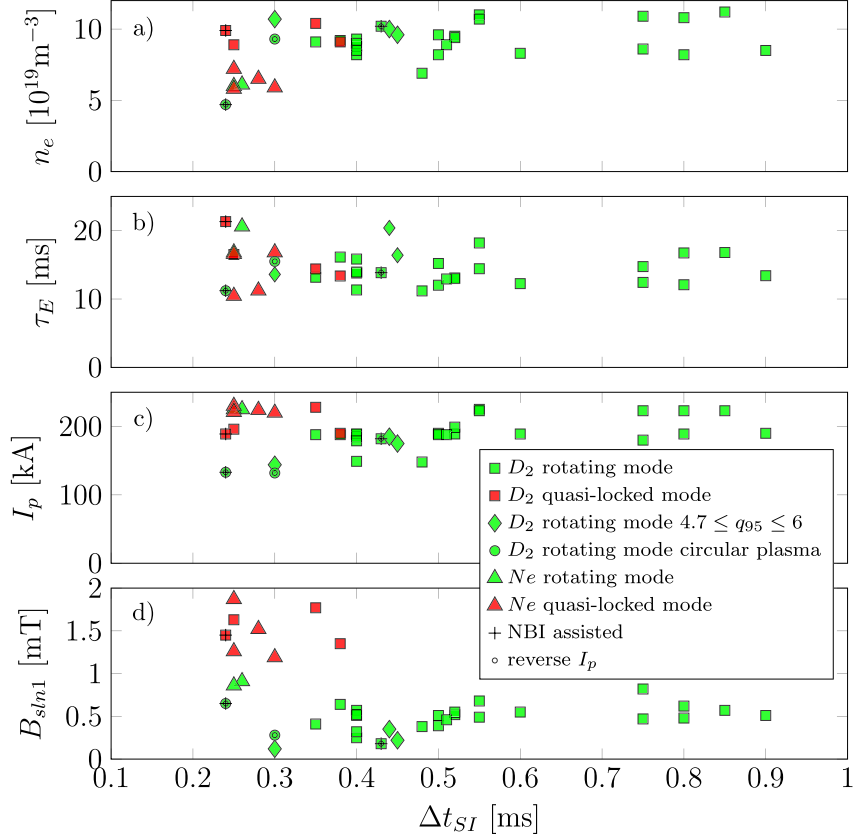

FIG. 4. (a) electron density, (b) energy confinement time, (c) plasma current, and

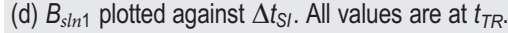

island growth. ${ }^{17,18}$ Also the discharges where the magnetic island becomes quasi-locked to the wall have shorter $\Delta t_{S I}$. These are also the cases where the magnetic island reached larger amplitude, as seen in Fig. 4(d) that shows the value of

$$
B_{s l n 1}=\frac{1}{2} \sqrt{\left(B_{s l}^{N W}-B_{s l}^{S E}\right)^{2}+\left(B_{s l}^{S W}-B_{s l}^{N E}\right)^{2}},
$$

where the superscripts indicate the intercardinal directions of each saddle loop coil. This behavior indicates that erosion of energy confinement develops faster, and the TQ is reached more quickly, when the magnetic island has a larger amplitude, or larger value of $B_{s \ln 1}$. The maximum and minimum values of $B_{s l n}$ differ by a factor of 3 , which is approximately also the same factor between the maximum and minimum values of $\Delta t_{S I}$. Major disruptions, i.e., TQ, are observed within these ranges of values.

The amplitude of the island at the onset of the SI, or when erosion of the energy confinement starts, is related to the rotation frequency of the magnetic island. This can be seen in Fig. 5(a), where the value of $B_{s l n 1}$ is plotted against the frequency of rotation of the $m / n=2 / 1$ magnetic island, $f_{2 / 1}$, both quantities are measured at $t_{T R}$. Figures $2(\mathrm{~d})-2(\mathrm{f})$ show how these two values were measured. The $f_{2 / 1}$ is the maximum frequency at $t_{T R}$ of the spectrogram of a saddle loop coil which, contrary to Mirnov coils, is less sensitive to the SI. At the transition time, the amplitude of the magnetic island varies inversely to its rotation frequency. In Fig. 5(b), $B_{s l n 1}$ is plotted in a vertical log scale, and Fig. 5(c) shows the product of $f_{2 / 1} \times B_{s \ln 1}$. From the collected data, it is not clear if both quantities are exponentially related or one is simply inversely proportional to the other.

The lines in Fig. 5(a) show the evolution of $B_{\operatorname{sln} 1}, 1.5 \mathrm{~ms}$ before $t_{T R}$. The solid (dashed) line is from pulse \#8945 (\#12748), which
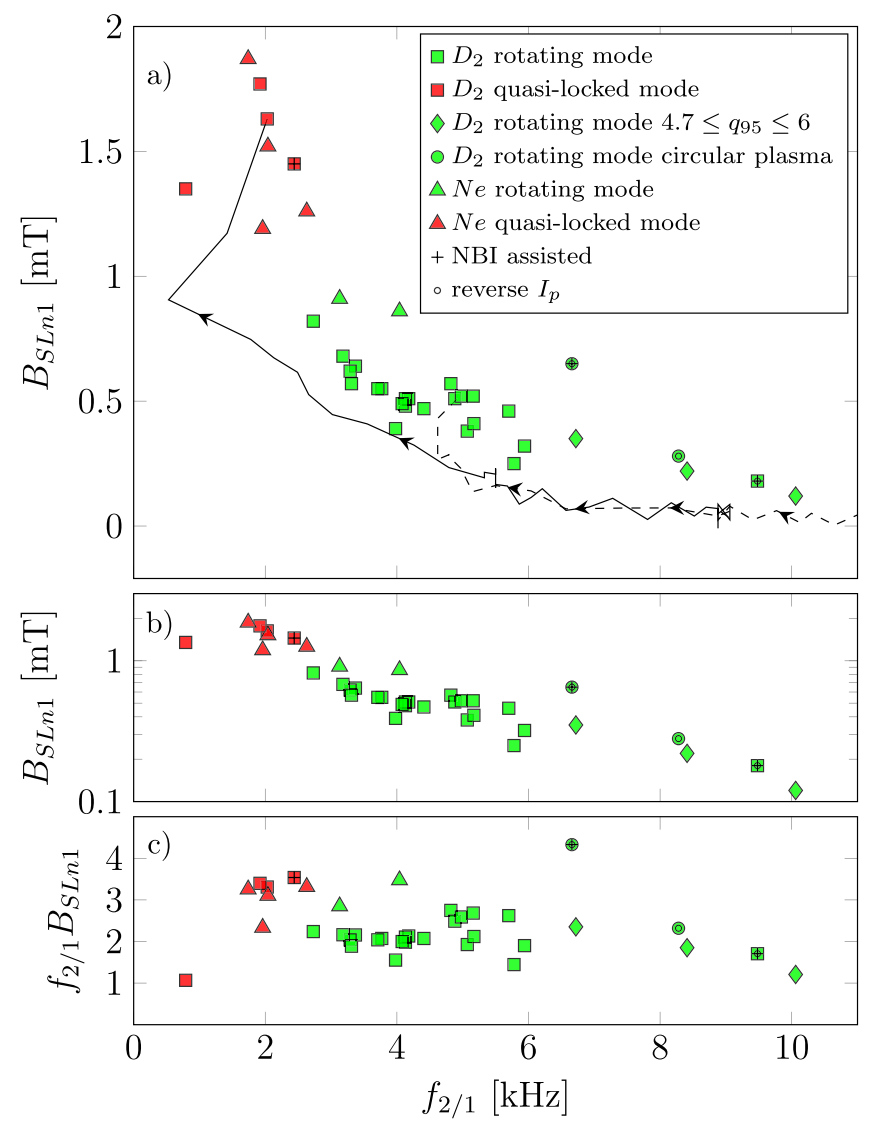

FIG. 5. (a) $B_{s \ln 1}$ against $t_{2 / 1}$ measured at $t_{T R}$ time for all the analyzed DLDs. The two lines show the evolution in time of $B_{s \ln 1}\left(f_{2 / 1}\right)$ for the discharges illustrated in Fig. 1, dashed line (solid line) for \#12748 (\#8945), during $1.5 \mathrm{~ms}$ before $t_{T R}$. Arrows show the time direction. (b) Same data in a linear-log scale. (c) $f_{2 / 1} \times B_{s l n}$ against $f_{2 / 1}$.

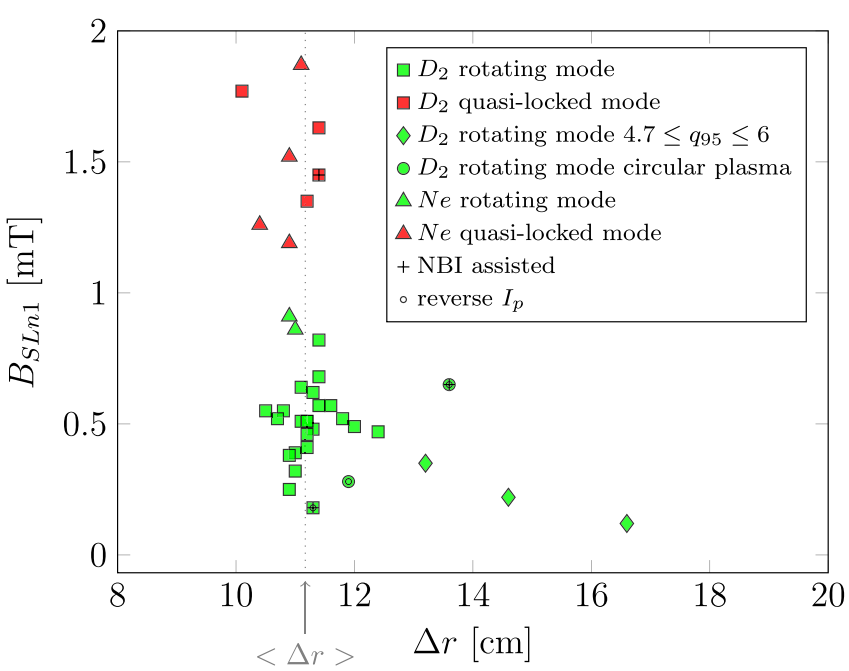

FIG. 6. $B_{s \ln 1}$ as a function of $\Delta r$, the distance of the $q=2$ surface to the vessel wall. 
also varies inversely with the rotation frequency except prior to the onset of the SI, when the magnetic island experiences a sudden increase in its rotation frequency and in its amplitude.

The data shows clearly that destruction of energy confinement can start at different values within $0.11 \mathrm{mT} \leq B_{\sin 1} \leq 1.87 \mathrm{mT}$, depending on the rotation frequency of the magnetic island at the onset of the SI. Destruction of energy confinement can start at a lower value of $B_{s \ln 1}$ if the island is rotating fast and vice versa for a slower rotating island. This is at odds with another study ${ }^{19}$ which found a distinct amplitude level when destruction of energy confinement starts. In that study, contrary to the work presented here, only locked magnetic islands were analyzed. Still considering just the cases where DLDs were preceded by quasi-locked modes, destruction of energy confinement can start at different values within $1.19 \mathrm{mT} \leq B_{\sin 1} \leq$ $1.87 \mathrm{mT}$, when the islands are rotating at a frequency of $\approx 2 \mathrm{kHz}$.

The discussed variations of $B_{\sin 1}$ are expected to reflect also the variations in island width, since the distance $\Delta r$ from the island rational surface at $q=2$ to the saddle loop coil position varied in a narrow range (see Fig. 6). In fact, excluding the few plasmas with larger $q_{95}$, the average distance was $\langle\Delta r\rangle=11.17 \mathrm{~cm} \pm 0.44 \mathrm{~cm}$.

\section{CONCLUSIONS}

This study of SI in COMPASS DLDs confirmed and considerably extended the observations previously made in JET. ${ }^{3}$ The range of different plasma types was expanded to D shaped (divertor) and circular (limiter) configuration, Deuterium and Neon gas puff, Ohmic and NBI assisted plasmas, and reversed $I_{p}$. In all of these cases, the SI was always found present at the onset of energy confinement erosion. In the majority of them, the precursor magnetic island was still rotating just before the onset of the SI, and in a minority of the cases, the island was quasi-locked to the vessel wall. Considering the quite distinct sizes of JET and COMPASS, it is expected that the SI is observed in all tokamak plasmas, including ITER. The duration of this erosion that culminates at the TQ was found to vary within $0.24 \mathrm{~ms} \leq \Delta t_{S I} \leq 0.9 \mathrm{~ms}$, with the cases with quasi-locked islands and Neon gas puff having the shortest durations. Independently of the values of global plasma parameters $n_{e}, \tau_{E}$, and $I_{p}, \Delta t_{S I}$ is shorter for larger values of $B_{s l n 1}$, so erosion of energy confinement develops faster, and the TQ is reached more quickly when the magnetic island has a larger amplitude.

At $t_{T R}$, the onset of SI, an inverse relation was also found between $B_{s \ln 1}$ and $f_{2 / 1}$, the magnetic island rotation frequency. This means that erosion of energy confinement can start at a different range of $B_{s l n} 1$ values, depending on what is the island rotation frequency at the onset of the SI. Erosion takes place either with smaller islands rotating faster or with larger islands rotating slower (or quasi-locked). This means that there is not a distinct island amplitude level at which destruction of energy confinement starts. The destruction starts only when the SI occurs, so the experimental evidence shows that it is the SI that triggers the disruption. A quantitative explanation of this inverse dependence between the island width and the island rotation frequency at the onset of the SI will enlighten understanding of the dynamics of the energy quench and of major disruptions. Meanwhile, the knowledge of this curve for a tokamak can provide a reliable trigger for major disruptions.

\section{SUPPLEMENTARY MATERIAL}

See the supplementary material with the same data as in Fig. 1 but with signal's amplitudes not standardized.

\section{ACKNOWLEDGMENTS}

This work was funded by Portuguese FCT - Fundação para a Ciência e Tecnologia, through Project No. UID/FIS/50010/2013 and Grant No. SFRH/BD/52415/2013 (PD-F APPLAuSE) and cofunded by Czech MEYS Project Nos. CZ.02.1.01/0.0/0.0/16_019/ 0000768 and LM2015045. The views expressed herein are the sole responsibility of the authors.

\section{REFERENCES}

${ }^{1}$ S. Mirnov, J. Wesley, N. Fujisawa, Y. Gribov, O. Gruber, T. Hender, N. Ivanov,

S. Jardin, J. Lister, F. Perkins et al., Nucl. Fusion 39, 2251 (1999).

${ }^{2}$ A. B. Rechester and M. N. Rosenbluth, Phys. Rev. Lett. 40, 38 (1978).

${ }^{3}$ F. Salzedas, Phys. Plasmas 18, 100701 (2011).

${ }^{4}$ S. I. W. Shah, F. Salzedas, J. Havlicek, J. Stöckel, and C. Silva, in 43rd EPS Conference on Plasma Physics, Leuven, Belgium, edited by P. Mantica, G. Giruzzi, M. Fajardo, and T. Gans (2016), Vol. 40A, p. P5.003.

${ }^{5}$ S. I. W. Shah, "Experimental study of secondary instability to $2 / 1$ magnetic island in COMPASS high density limit plasmas," Ph.D. thesis (Universidade de Lisboa-Instituto Superior Técnico, Lisboa, 2018).

${ }^{6}$ Y. Ishii, M. Azumi, and A. I. Smolyakov, Nucl. Fusion 47, 1024 (2007).

${ }^{7}$ N. F. Loureiro and D. A. Uzdensky, Plasma Phys. Controlled Fusion 58, 014021 (2016).

${ }^{8}$ T. Markovic, Y. Q. Liu, P. Cahyna, R. Pánek, M. Peterka, M. Aftanas, P. Bílková, P. Bohm, M. Imríšek, P. Háček et al., Nucl. Fusion 56, 092010 (2016).

${ }^{9}$ V. G. Merezhkin, Sov. J. Plasma Phys. 4, 152 (1978).

${ }^{10}$ O. Klüber, H. Zohm, H. Bruhns, J. Gernhardt, A. Kallenbach, and H. P. Zehrfeld, Nucl. Fusion 31, 907 (1991).

${ }^{{ }^{11}}$ T. H. Jensen and M. S. Chu, J. Plasma Phys. 30, 57 (1983).

${ }^{12}$ M. F. F. Nave and J. Wesson, Nucl. Fusion 30, 2575 (1990).

${ }^{13}$ R. Fitzpatrick, Nucl. Fusion 33, 1049 (1993).

${ }^{14}$ R. Fitzpatrick, R. J. Hastie, T. J. Martin, and C. M. Roach, Nucl. Fusion 33, 1533 (1993).

${ }^{15}$ I. H. Hutchinson, Plasma Phys. Controlled Fusion 43, 145 (2001).

${ }^{16}$ H. Zohm, A. Kallenbach, H. Bruhns, G. Fussmann, and O. Klüber, Europhys. Lett. 11, 745 (1990).

${ }^{17}$ F. Salzedas, F. C. Schüller, and A. A. M. Oomens, Phys. Rev. Lett. 88, 075002 (2002).

${ }^{18}$ D. A. Gates, D. P. Brennan, L. Delgado-Aparício, Q. Teng, and R. B. White, Phys. Plasmas 23, 056113 (2016).

${ }^{19}$ P. C. de Vries, G. Pautasso, E. Nardon, P. Cahyna, S. Gerasimov, J. Havlicek, T. C. Hender, G. T. A. Huijsmans, M. Lehnen, M. Maraschek et al., Nucl. Fusion 56, 026007 (2016). 